\title{
Advances With Non-coding RNAs in Neuropathic Pain
}

\author{
Cheng $\mathrm{Hu}^{\dagger}$, Menglin $\mathrm{He}^{\dagger}$, Qian Xu and Weiqian Tian* \\ Affiliated Hospital of Nanjing University of Chinese Medicine, Jiangsu Province Hospital of Chinese Medicine, Nanjing, China
}

\section{OPEN ACCESS}

Edited by:

Francesco Napolitano,

King Abdullah University of Science and Technology, Saudi Arabia

Reviewed by:

Estela Maria Bruxel,

State University of Campinas, Brazil

Songxue Su,

Zhengzhou University, China

*Correspondence:

Weiqian Tian

yfy0066@njucm.edu.cn

tThese authors have contributed

equally to this work

Specialty section:

This article was submitted to

Neurogenomics,

a section of the journal

Frontiers in Neuroscience

Received: 20 August 2021

Accepted: 29 October 2021

Published: 23 December 2021

Citation:

Hu C, He M, Xu Q and Tian W (2021) Advances With Non-coding

RNAs in Neuropathic Pain.

Front. Neurosci. 15:760936.

doi: 10.3389/fnins.2021.760936
Neuropathic pain (NP) is one of the most common types of clinical pain. The common causes of this syndrome include injury to the central or peripheral nervous systems and pathological changes. NP is characterized by spontaneous pain, hyperalgesia, abnormal pain, and paresthesia. Because of its diverse etiology, the pathogenesis of NP has not been fully elucidated and has become one of the most challenging problems in clinical medicine. This kind of pain is extremely resistant to conventional treatment and is accompanied by serious complications. Non-coding RNAs (ncRNAs), such as microRNAs (miRNAs), long non-coding RNAs (IncRNAs), and circular RNAs (circRNAs), contribute to diverse biological processes by regulating the expression of various mRNAs involved in pain-related pathways, at the posttranscriptional level. Abnormal regulation of ncRNAs is closely related to the occurrence and development of NP. In this review, we summarize the current state of understanding of the roles of different ncRNAs in the development of NP. Understanding these mechanisms can help develop novel therapeutic strategies to prevent or treat chronic pain.

Keywords: neuropathic pain, non-coding RNAs, miRNA, IncRNA, circRNA

\section{INTRODUCTION}

The International Association for the Study of Pain Taxonomy defines neuropathic pain (NP) as "pain caused by a lesion or disease of the somatosensory nervous system" (WiderstromNoga, 2017). The pathogenesis of NP includes peripheral and central mechanisms. The peripheral mechanism includes the abnormal discharge of injured peripheral afferent fibers, the electrical and

Abbreviations: NP, Neuropathic pain; ncRNAs, non-coding RNAs; lncRNAs, long non-coding RNAs; DRG, dorsal root ganglion; UTR, untranslated region; pSNL, partial sciatic nerve ligation; SNI, spared nerve injury; CXCR4, chemokine CXC receptor 4; TXNIP, thioredoxin-interacting protein; NLRP3, NOD-like receptor protein 3; CCI, chronic constriction injury; HMGB1, high-mobility group box 1 protein; CXCL13, C-X-C motif chemokine 13; Cav1.2-LTC, L-type calcium channel; SIRT1, sirtuin1; Tregs, regulatory T cells; SDH, spinal dorsal horn; IRAK1, inhibiting interleukin-1 receptorassociated kinase 1; TRAF6, tumor necrosis factor receptor-associated factor 6; TIR, toll/interleukin-1 receptor; STAT3, signal transducer and activator of transcription 3; SOCS3, suppressor of cytokine signaling 3; NF-kB, nuclear factor-kappa B; p38 MAPK, p38 mitogen-activated protein kinase; SOCS1, suppressor of cytokine signaling 1; SN, sciatic nerve; BDNF, brainderived neurotrophic factor; $\mathrm{Cx} 43$, connexin 43 ; $\mathrm{BAMBI}$, bone morphogenetic protein and membrane-bound inhibitor; TGF- $\beta$, transforming growth factor- $\beta$; ZEB1, zinc finger E box binding protein-1; MeCP2, Methyl CpG binding protein 2; NOX4, NADPH oxidase 4; SCI, spinal cord injury; TLR8, toll-like receptor 8; ERK, extracellular regulated protein kinases; DNP, diabetic neuropathic pain; TRPV1, transient receptor potential vanilloid type 1; P2 $\times 7$, P2X purinoceptor; SGCs, satellite glial cells; L5, lumbar 5; CDK6, cyclin-dependent kinase 6; CRNDE, colorectal neoplasia differentially expressed; XIST, X inactive specific transcript; TNFAIP1, tumor necrosis factor alpha-induced protein 1; COX-2, cyclooxygenase-2; TNF- $\alpha$, tumor necrosis factor- $\alpha$; IL-1 $\beta$, MALAT1, interleukin- $1 \beta$; metastasis-associated lung adenocarcinoma transcript 1; NAT, natural antisense transcript; circRNAs, circular RNAs; PHN, postherpetic neuralgia; PDK4, pyruvate dehydrogenase kinase 4; RAP1A, Ras-associated protein 1A; SLC17A6, [solute carrier family 17 (sodium-dependent inorganic phosphate cotransporter), member 6]; NEAT1, nuclear paraspeckle assembly transcript 1; AQP4, aquaporin-4; MIP-1 $\alpha$, macrophage inflammatory protein-1 alpha and CCR1, C-C chemokine receptor type 1; AMPK, AMP-activated protein kinase; EZH2, enhancer of zeste homolog 2; VEGFB, vascular endothelial growth factor B; ENO1, enolase 1; LPAR3, lysophosphatidic acid receptor 3; BACE1, $\beta$-site amyloid precursor protein-cleaving enzyme 1 . 
sympathetic sensory coupling induced by the mixed transmission of neurons, and the increased excitability of adjacent undamaged fibers. The central mechanism includes the sensitization of spinal dorsal horn (SDH) neurons, decline of central inhibitory intermediate neurons, the sprouting of $\mathrm{A}_{\beta}$ fibers, activation of the descending facilitation system, and sensitization and activation of glial cells in the higher brain center. Many kinds of peripheral or central nervous system disorders can cause chronic pain. Diabetes, hypothyroidism, uremia, nutritional deficiency, and chemotherapy drugs (vincristine, paclitaxel, cisplatin, etc.) can lead to neuralgia, Guillain-Barre syndrome, postherpetic neuralgia, progressive neuromuscular atrophy, complex regional pain syndrome type $I$, and ischemic neuropathy. The comorbidities in patients with NP include poor sleep, depression, and anxiety. Thus, NP greatly impairs quality of life and has a high economic impact on society. Despite the widespread interest, the pathogenesis of NP is not well understood to date, which in turn has resulted in the lack of effective clinical treatment options. It has become a difficult and hot topic in pain research (von Hehn et al., 2012; Yan et al., 2018a).

NP has become a universal healthcare issue affecting a wide range of people across the world. In the United States, NP costs an estimated $\$ 600$ billion a year in healthcare and productivity losses (Gilron and Dickenson, 2014; Holmes, 2016). At present, the commonly used clinical treatment methods include local nerve block, sympathetic block, physiotherapy, opioid anesthetics, antiinfectives, antidepressants, and/or anxiolytics. In spite of these various options, pain management remains a challenge. To develop newer and better treatment strategies, it is necessary to gain a better understanding of the molecular mechanisms that lead to NP (Yan et al., 2017).

RNA has always been considered as a simple and intermediate component of gene expression, because it is transcribed from DNA and then translated into proteins in cells. However, the mammalian genome encodes not only protein-coding RNA but also a large number of non-coding RNAs (ncRNAs) (Lutz et al., 2014). ncRNAs play an important role in physiological and pathological NP processing. Specific processes within the cellular components of the NP triad that includes neurons, immune cells, and glia as well as the communication pathways among them are controlled by specific microRNAs (miRNAs) (Plassais et al., 2016; Liu et al., 2017; Shao et al., 2018; Vieira et al., 2018; Kalpachidou et al., 2020). A few studies have shown that miRNAs modulate inflammatory and NP conditions and therefore can be used as biomarkers of these conditions (Andersen et al., 2014; Gaudet et al., 2018). Long non-coding RNAs (lncRNAs) and circular RNAs (circRNAs) regulate mRNA expression in the progression of NP via their cross talk with miRNAs (Song et al., 2020).

In this article, we review the existing evidence for the changes in the three types of ncRNAs-miRNA, lncRNA, and circRNAin pain-related regions after nerve injury. We also discuss how peripheral noxious stimuli induce such changes and explore potential mechanisms of how expressional changes in dorsal root ganglion (DRG), injured nerve, or spinal cord dorsal horn ncRNAs contribute to the development and persistence of NP. The known ncRNAs play a key role in gene regulation. Harmful peripheral stimuli can cause changes in the expression of ncRNAs which are related to hyperalgesia in NP (Bali and Kuner, 2014).

\section{MICRORNAS IN NEUROPATHIC PAIN}

MiRNAs are small (21-23 nucleotides) ncRNA molecules that bind to the $3^{\prime}$ untranslated region (UTR) of the target mRNA at a specific sequence and act as posttranscriptional regulators of gene expression. The regulatory mechanisms include silencing mRNA expression or degrading the transcript in various biological processes or states (Aldrich et al., 2009). Mounting evidence shows that miRNA is strongly associated with pain pathways from primary afferent pain receptors, DRG, spinal cord, and brain regions in different NP models (Sun et al., 2012; Sakai et al., 2013; Park et al., 2014; Pan et al., 2016; Peng et al., 2017). Some cases of neuropathic, inflammatory, and cancer pain can be prevented or reversed via the regulation of miRNA expression (Bali et al., 2013; Pan et al., 2014; Jiang et al., 2016). The possible role of miRNAs in gene regulation relative to NP is discussed below.

In partial sciatic nerve ligation ( $\mathrm{pSNL}$ ) induced $\mathrm{NP}$, the expression of chemokine CXC receptor 4 (CXCR4) was higher and miRNA-23a-3p (miR-23a) expression was significantly lower in spinal glial cells. MiR-23a can bind directly to the $3^{\prime}$ UTR of CXCR4 mRNA. Knockdown of miR-23a or overexpression of CXCR4 in mice can increase the thioredoxin-interacting protein (TXNIP), which is related to the induction of NODlike receptor protein 3 (NLRP3) inflammasome in pSNL mice. Inhibition of TXNIP can reverse pain behaviors induced by pSNL. In short, miR-23a regulates NP in pSNL mice by directly targeting CXCR4, via the TXNIP/NLRP3 inflammasome axis (Pan et al., 2018). In mice with chronic constriction injury (CCI), miR-381 can inhibit the development of NP by targeting the high-mobility group box 1 protein (HMGB1) and CXCR4 (Zhan et al., 2018). Peripheral nerve injury activates the expression and release of $\mathrm{C}-\mathrm{X}-\mathrm{C}$ motif chemokine 13 (CXCL13) from spinal cord neurons to have an effect on the CXCR5 in astrocytes. The induced astrocytes activate to maintain NP. MiR-186-5p negatively regulates CXCL13. The downregulation of miR-186$5 \mathrm{p}$ in spinal cord neurons in SNL leads to the upregulation of CXCL13, which leads to NP (Jiang et al., 2016).

Cav1.2 consists of the L-type calcium channel (Cav1.2LTC) and plays a key role in chronic NP. MiR-103 can simultaneously affect the expression of the three subunits forming Cav1.2-LTC in a novel integrative regulation. MiR103 plays a bidirectional and integrated regulatory role in Cav1.2-LTC, which is likely to be the primary cause of chronic pain sensitization. In fact, downregulation of miR103 levels can induce hyperalgesia in animals (Favereaux et al., 2011). MiR-17-92, a miRNA cluster with six different elements, cannot only downregulate the expression of potassium channels but also reduce extracellular potassium currents, especially A-type currents. A combination of potassium channel regulators can relieve the mechanical allodynia caused by nerve injury or overexpression of miR-17-92. The miR-17-92 cluster seems to co-regulate the function of multiple voltage-gated 
potassium channel subunits (Sakai et al., 2017). MiR-7a is a pivotal functional miRNA, which maintains the stage of NP in rats by regulating the excitability of neurons. In the late stage of $\mathrm{NP}$, miR-7a is a noticeable change miRNA in the injury DRG. The downregulation of miR-7a in itself is not sufficient to lead to pain-related behaviors in rats. MiR-7a targets the $\beta 2$ subunit of the voltage-gated sodium channel. A decrease in miR-7a leads to the increase in $\beta 2$ subunit protein expression. The increased expression of the $\beta 2$ subunit results in long-term hyperactivity of the injured neurons and persistence of pain behaviors (Sakai et al., 2013). In SNL-induced NP, miR-30b can directly target SCN3A $3^{\prime} \mathrm{UTR}$, alleviating NP mainly via inhibiting the expression of voltage-gated sodium channel Nav1.3 in DRG neurons and spinal cord (Su et al., 2017).

SNL results in the downregulation of miRNAs such as miR183 family in ipsilateral lumbar 5 (L5) DRG. In addition, the nerve injury leading to mechanical hypersensitivity is accompanied by the general redistribution of miRNA around DRG neurons with and without myelin sheath (Aldrich et al., 2009). The expression levels of miR-124a and miR-155 were higher and the histone deacetylase sirtuin1 (SIRT1) levels were lower in NP patients compared to those in healthy volunteers. In fact, the two miRNAs were negatively correlated with SIRT1 transcription level and were found to be direct inhibitors of SIRT1 in human CD4 + cells. SIRT1, targeted by these two miRNAs, can increase the expression level of Foxp3, which leads to an increase in anti-inflammatory regulatory $\mathrm{T}$ cells (Tregs) (Heyn et al., 2016). It was found that in DRG, IL-6 increased the expression of miR-21, miR-431, and miR-511-3p. The expression of miR-21 in exosomes extracted from blood was higher after spared nerve injury (SNI), which may play a partial role in NP (Hori et al., 2016). There is evidence which shows that miRNA-146a-5p in DRG and SDH can regulate CCI-induced NP by inhibiting interleukin-1 receptor-associated kinase 1 (IRAK1) and tumor necrosis factor receptor-associated factor 6 (TRAF6) in toll/interleukin-1 receptor (TIR) signaling pathway (Wang Z. et al., 2018). MiR-146a-5p can alleviate NP by inhibiting TRAF6 and its downstream JNK/CCL2 signal pathway (Lu et al., 2015). MiR-218 negatively mediates the signal transducer and activator of transcription 3 (STAT3) signaling pathway via regulating the suppressor of cytokine signaling 3 (SOCS3) expression, and miR-221 inhibits nuclear factorkappa B (NF-kB) and p38 mitogen-activated protein kinase (p38MAPK) signaling pathway by regulating the SOCS1 level to alleviate CCI-induced NP and neuroinflammation (Li and Zhao, 2016; Xia et al., 2016). MiR-223 can inhibit the activity of NLRP3 inflammasomes by downregulating NLRP3, which in turn reduces morphine tolerance in rats with CCI-induced NP. MiR-34c can inhibit the development of NP primarily by inhibiting NLRP3-regulated neuroinflammation (Xie X.J. et al., 2017; Xu et al., 2019). MiR-26a-5p regulates the development of NP by targeting MAPK6 in CCI-treated rats (Zhang et al., 2018a). In rats with NP, CCI resulted in a time-dependent downregulation of miRNA-1 in sciatic nerve (SN), while miRNA-1-targeted protein brain-derived neurotrophic factor (BDNF), connexin $43(\mathrm{Cx} 43)$ in $\mathrm{SN}$, and DRG were upregulated (Neumann et al., 2015).
In rats with $\mathrm{SN}$ injury, the expression of miR-30c-5p positively correlated with NP. MiR-30c-5p is related to the bone morphogenetic protein and membrane-bound inhibitor (BAMBI), a transforming growth factor- $\beta$ (TGF- $\beta$ ) pseudo receptor (Tramullas et al., 2018). The abnormal expression of miR-132-3p is strongly associated with human NP. In the SNI model, miR-132-3p levels were increased leading to the upregulation of AMPA receptor subunits GluA1 and GluA2 in the spinal cord (Leinders et al., 2016).

Upregulation of miR-200b and miR-429 expression in CCI mice can inhibit neuroinflammation by inhibiting inflammatory cytokines. MiR-200b/miR-429 can be an important regulator of NP by targeting zinc finger $\mathrm{E}$ box binding protein-1 (ZEB1) (Yan et al., 2018c). In the CCI rats, miR-378 was downregulated, and the overexpression of miR-378 provided significant pain relief. Enhancer of zeste homolog 2 (EZH2) is the downstream gene of $\mathrm{miR}-378$ and is negatively regulated by miR-378 (Gao et al., 2021). The methyl CpG-binding protein 2 (MeCP2) plays a key role in neuron differentiation, neural development, and synaptic plasticity by modulating CREB-miR132 to relieve acute and chronic pain (Zhang et al., 2015). Inhibition of the mTOR or VEGF receptor can significantly reduce NP, and the upregulation of miR-183 can suppress the AMPA receptor by inhibiting the mTOR/VEGF pathway (Xie X. et al., 2017). In CCI mice, overexpression of miR233 inhibits the polarization and recruitment of macrophages via the NLRP3/IL-1 $\beta$ pathway to ameliorate NP (Zhu et al., 2021). In spinal cords of rats with CCI-induced NP, miR-122$5 p$ expression was decreased. Overexpression of miR-122-5p can prevent NP behavior. Pyruvate dehydrogenase kinase 4 (PDK4) is a downstream protein, and inhibition of its expression by miR122-5p attenuates NP (Wan et al., 2021). MiR-142-3p expression was higher, and AC9 expression was lower in rats with CCIinduced NP. MiR-142-3p inhibition could increase the expression of AC9 and CAMP and further increase the activity of AMPK (Li et al., 2021).

In the DRG of oxaliplatin-induced NP rats, miR-30b$5 \mathrm{p}$ expression decreased meanwhile the mRNA and protein expression levels of $\mathrm{Na}_{\mathrm{v}} 1.6$ increased. MiR-30b-5p contributes to NP through $\mathrm{Na}_{\mathrm{v}} 1.6$ downregulation (Li L. et al., 2019). MiR$15 \mathrm{~b}$ was increased in the oxaliplatin-induced NP, $\beta$-site amyloid precursor protein-cleaving enzyme 1 (BACE1) was a target of miR-15b, and miR-15b and BACE1 were negatively correlated in NP (Ito et al., 2017). It was found that NP caused by paclitaxel or SNL significantly damaged the GABAergic synaptic function of $\mathrm{SDH}$ neurons by reducing the expression of GAD67. The expression levels of miR-500 increased in NP and regulated GAD67 by targeting specific sites of the GAD1 gene in the dorsal horn. Downregulation of miR-500 could rescue the GABAergic synapses in the SDH neurons and attenuate the sensitized pain behavior in rats with NP (Huang et al., 2016). Intrathecal injection of miRNA-138 can alleviate NP in rats with a pSNL, which may be achieved by suppressing the toll-like receptor 4 (TLR4) and macrophage inflammatory protein-1 alpha (MIP$1 \alpha) / C-C$ chemokine receptor type 1 (CCR1) signaling pathways (Jin et al., 2021). The downregulation of NADPH oxidase 4 (NOX4) caused by miR-23b can effectively induce TXNL1, GPX3, 
and SEPN1 gene expression after spinal cord pain induction. MiR-23b plays an important role in the improvement of NP caused by spinal cord injury (SCI) by inactivating NOX4. In animals with NP, the exogenous expression of miR-23b and NOX4 antibody effectively reduced pain symptoms and increased the survival of GABAergic neurons and the recovery of GAD expression in SCI. The expression of exogenous miR$23 \mathrm{~b}$ in neuropathic lesions can effectively control inflammatory symptoms, including microglial infiltration and inflammatory factor secretion, and reduce ROS-mediated GABAergic nerve cell death (Im et al., 2012). MiR-183 modulates the expression of MAP3K4, which in turn leads to the downregulation of inflammatory factors, and cyclooxygenase-2 (COX-2) which slows NP progression. In summary, the study stated that miR-183 was a part of the negative regulatory effector group, which could alleviate NP by targeting MAP3K4 (Huang and Wang, 2020). MiR-21 can interact with toll-like receptor 8 (TLR8) in lysosome as an endogenous ligand, inducing extracellularly regulated protein kinase (ERK) activation and production of inflammatory mediators. It further enhances the excitability of neurons and contributes to the persistence of NP (Zhang et al., 2018c).

In diabetic neuropathic pain (DNP), the miR-190a-5p level was decreased and SLC17A6 [the solute carrier family 17 (sodium-dependent inorganic phosphate cotransporter), member 6] was increased in the spinal tissue compared to that in the control group. Upregulation of miR-190a-5p and inhibition of SLC17A6 could significantly weaken the painrelated behavior and reduce IL- $1 \beta$ and IL- 6 levels in DNP (Yang et al., 2017). Inhibition of miR-221 can reduce pain and decrease the expression of pain-related factors (bradykinin, prostaglandin E2, IL-6, IL-1 $\beta$, and tumor necrosis factor alpha) through targeting SOCS3 in the DNP rat model (Wu et al., 2021). MiR-590-3p inhibits $\mathrm{T}$ cell infiltration by targeting Ras-associated protein 1A (RAP1A), thus ameliorating diabetic peripheral NP in animal models (Wu et al., 2020).

In some commonly used NP models, specific miRNAs are upregulated or downregulated along the pain pathway. The above part summarizes the imbalance of miRNA in pain model tissues and the role of related target genes in the mechanism of NP. MiRNAs associated with NP are listed in Table 1.

\section{LONG NON-CODING RNA IN NEUROPATHIC PAIN}

LncRNA is a new kind of functional RNA discovered in the past decade. It is over 200 nucleotides long and is generally believed to play a crucial role in the regulation of gene expression. Its biological and molecular mechanisms are diverse and complex, which need further study (Zhang et al., 2018b). Rapidly accumulating evidence points out that close to $40 \%$ of IncRNA exists exclusively in the nervous system (Wu W. et al., 2019). LncRNAs have recently been found to be key modulators of neuronal functions, especially in NP (Li Z. et al., 2019).

In the SDH of mice with DNP, 1,481 differentially expressed (DE) lncRNAs and 1,096 DE mRNAs have been identified.
It has been found that 289 neighboring and 57 overlapping IncRNA-mRNA pairs, including ENSMUST00000150952-Mbp and AK081017-Usp15, may be involved in DNP pathogenesis (Du et al., 2019). Liu C. et al. (2018) found that ERK and p38 MAPK signaling pathways may be involved in the processes by which lncRNA BC168687 siRNA alleviates DNP mediated by transient receptor potential vanilloid type 1 (TRPV1). Another study found that inhibition of lncRNA BC168687 expression may downregulate $\mathrm{P} 2 \mathrm{X}$ purinoceptor $(\mathrm{P} 2 \times 7)$ receptor expression in satellite glial cells (SGCs) induced by high glucose and high free fatty acid environment (Liu C.L. et al., 2018). In type 2 DNP rats, siRNA of IncRNA NONRATT021972 can reduce the expression of $\mathrm{P} 2 \times 7 \mathrm{mRNA}$ and protein in DRG and inhibit the activation of SGCs. In addition, NONRATT021972 siRNA treatment reduced the release of tumor necrosis factor$\alpha$ (TNF- $\alpha$ ), thereby inhibiting the excitability of DRG neurons and reducing NP (Liu et al., 2016). The expression of lncRNA uc 0.48 + was increased in the DRG of DNP rats, and serum of diabetic patients. The $\mathrm{P} 2 \times 3$ protein and mRNA in DRG of diabetic rats were increased while uc $0.48+$ siRNA treatment reverses the trend. Phosphorylation and activation of ERK1/2 also decreased. Therefore, uc $0.48+$ siRNA treatment can alleviate DNP by inhibiting P2 $\times 3$ receptor-mediated excitatory transfer in DRG (Wang et al., 2016). In the lumbar SDH of DNP mice, miR-193a was downregulated and HMGB1 expression was upregulated. Overexpression of miR-193a can inhibit HMGB1 expression and alleviate NP (Wu B. et al., 2019). LncRNA, NONRATT021972 increased in type 2 diabetes and was positively associated with NP score (Yu et al., 2017). In trigeminal ganglia, $\operatorname{lncRNA}$ uc $0.48+$ can interact with the $\mathrm{P} 2 \times 7$ receptor, upregulate $\mathrm{P} 2 \times 7$ receptor expression, enhance ERK1/2 phosphorylation, and participate in pain transmission (Xiong et al., 2019). LncRNA, MRAK009713 could play a positive role in NP in rats through regulating the expression and function of the $\mathrm{P} 2 \times 3$ receptor (Li et al., 2017).

In the SNL-induced NP mice, the DRGs of L5 were taken for microarray analysis of IncRNAs. From the fourth day of SNL treatment, H19 IncRNA increased significantly in L5 DRG and was detected mainly in non-neuronal cells. Consistent with this finding, the expression of H19 was upregulated in Schwann cells isolated from peripheral nerves, which might be related to NP (Iwasaki et al., 2019). In SNL model rats, IncRNA, PKIA-AS1 was significantly upregulated, while the inhibition of PKIA-AS1 slowed the progression of NP. In addition, overexpression of PKIA-AS1 can remarkably induce NP-like symptoms in uninjured rats. PKIA-AS1 can mediate SNL-induced NP through regulating the expression and function of cyclin-dependent kinase 6 (CDK6), which is essential for the occurrence and progression of neuroinflammation and NP. Therefore, PKIA-AS1 is expected to become a new therapeutic target for neuroinflammation associated with NP (Hu et al., 2019). The lncRNA Kcna2 antisense RNA, voltage-dependent potassium channel mRNA, can be regulated by transcriptional activation like mRNA. The upregulation of Kcna2 antisense RNA caused by nerve injury targets myeloid zinc finger protein 1 , a transcription factor that binds to the Kcna2 antisense RNA gene promoter (Zhao et al., 2013). 
TABLE 1 | miRNAs associated with neuropathic pain.

\begin{tabular}{|c|c|c|c|c|c|}
\hline Models & miRNAs & Expression & Tissue & Target gene & References \\
\hline pSNL & miR-23a-3p & $\downarrow$ & Spinal glial cells & CXCR4 TXNIP/NLRP3 & Pan et al., 2018 \\
\hline $\mathrm{CCl}$ & miR-381 & $\uparrow$ & Spinal cord tissues & HMGB1/CXCR4 & Zhan et al., 2018 \\
\hline SNL & $\mathrm{miR}-186-5 \mathrm{P}$ & $\downarrow$ & Spinal astrocyte & CXCL13 & Jiang et al., 2016 \\
\hline SNL & miR-103 & $\downarrow$ & Spinal cord & Cav1.2-LTC & Favereaux et al., 2011 \\
\hline SNL & miR-17-92 & $\uparrow$ & $\mathrm{DRG}$ & $\begin{array}{l}\text { Voltage-gated potassium } \\
\text { channel }\end{array}$ & Sakai et al., 2017 \\
\hline SNL & $\mathrm{miR}-7 \mathrm{a}$ & $\downarrow$ & $\mathrm{DRG}$ & Voltage-gated sodium channel & Sakai et al., 2013 \\
\hline SNL & miR-30b & $\downarrow$ & DRG/spinal cord & $\begin{array}{l}\text { Voltage-gated sodium channel } \\
\text { Nav1.3 }\end{array}$ & Su et al., 2017 \\
\hline NP patients & miR-124a, miR-155 & $\uparrow$ & Blood samples & $\mathrm{SIRT1}$ & Heyn et al., 2016 \\
\hline SNL & miR-183 & $\downarrow$ & L5 DRG & $\begin{array}{l}\text { T-Cell intracellular antigen } 1 \\
(\mathrm{TIA}-1)\end{array}$ & Aldrich et al., 2009 \\
\hline SNL & miR-21, miR-431, miR-511-3p & $\uparrow$ & $\mathrm{DRG}$ & $\mathrm{IL}-6$ & Hori et al., 2016 \\
\hline $\mathrm{CCl}$ & $m i R-146 a-5 p$ & $\uparrow$ & DRG/SDH & IRAK1/TRAF6 & Wang Z. et al., 2018 \\
\hline SNL & $\operatorname{miR}-146 a-5 p$ & $\uparrow$ & Spinal cord & TRAF6/JNK & Lu et al., 2015 \\
\hline $\mathrm{SCl}$ & miR-218 & $\downarrow$ & Spinal cord & SOCS3/p-P38 & Li and Zhao, 2016 \\
\hline $\mathrm{CCl}$ & miR-221 & $\uparrow$ & Spinal cord & SOCS1 & Xia et al., 2016 \\
\hline $\mathrm{CCl}+$ tolerance morphine & miR-223 & $\downarrow$ & Spinal cord & NLRP3 & Xie X.J. et al., 2017 \\
\hline $\mathrm{CCl}$ & $\mathrm{miR}-34 \mathrm{c}$ & $\downarrow$ & Spinal cord & NLRP3 & Xu et al., 2019 \\
\hline $\mathrm{CCl}$ & miR-26a-5p & $\downarrow$ & Spinal cord & MAPK6 & Zhang et al., 2018a \\
\hline $\mathrm{CCl}$ & $\operatorname{miR}-1$ & $\downarrow$ & SN/DRG & $\mathrm{C} \times 43 / \mathrm{BDNF}$ & Neumann et al., 2015 \\
\hline SNI & miR-30c-5p & $\uparrow$ & $\begin{array}{l}\text { Spinal } \\
\text { cord/DRG/plasma/fluid } \\
\text { cerebrospinal }\end{array}$ & $\mathrm{BAMBI}$ & Tramullas et al., 2018 \\
\hline DNP & miR-190a-5p & $\downarrow$ & Spinal dorsal horn & SLC17A6 & Yang et al., 2017 \\
\hline DNP & miR-193a & $\downarrow$ & Spinal dorsal horn & HMGB1 & Wu B. et al., 2019 \\
\hline DNP & miR-221 & $\uparrow$ & Serum & socs3 & Wu et al., 2021 \\
\hline DPNP & miR-590-3p & $\downarrow$ & $\mathrm{DRG} / \mathrm{T}$ cells & RAP1A & Wu et al., 2020 \\
\hline $\mathrm{SCl}$ & miR-130a-3p & $\uparrow$ & Spinal cord & IGF-1/IGF-1R & Yao et al., 2021 \\
\hline $\mathrm{CCl}$ & miR-378 & $\downarrow$ & Spinal cord & $\mathrm{EZH} 2$ & Gao et al., 2021 \\
\hline pSNL & $\mathrm{miR}-138$ & $\uparrow$ & Spinal cord & TLR4 MIP-1a/CCR1 & Jin et al., 2021 \\
\hline $\mathrm{CCl}$ & miR-223 & $\downarrow$ & Spinal cord & NLRP3/IL-1 $\beta$ & Zhu et al., 2021 \\
\hline $\mathrm{CCl}$ & miR-122-5p & $\downarrow$ & Spinal cord & PDK4 & Wan et al., 2021 \\
\hline $\mathrm{CCl}$ & miR-142-3p & $\uparrow$ & Sciatic nerve & CAMP/AMPK & Li et al., 2021 \\
\hline NP patients/SNI & miR-132-3p & $\uparrow$ & $\begin{array}{l}\text { White blood } \\
\text { cells/SN/DRG/Spinal } \\
\text { cord }\end{array}$ & GluA1/GluA2 & Leinders et al., 2016 \\
\hline $\mathrm{CCl}$ & miR-200b/miR-429 & $\uparrow$ & $\begin{array}{l}\text { Microglia from the } \\
\text { Spinal cord }\end{array}$ & ZEB1 & Yan et al., 2018c \\
\hline SNI & CREB-miR-132 & $\downarrow$ & Spinal cord & $\mathrm{MeCP} 2$ & Zhang et al., 2015 \\
\hline $\mathrm{CCl}$ & miR-183 & $\downarrow$ & Spinal cord horn & mTOR/VEGF & Xie X. et al., 2017 \\
\hline Oxaliplatin-induced NP & miR-30b-5p & $\downarrow$ & $\mathrm{DRG}$ & $\mathrm{Na}_{\mathrm{v}} 1.6$ & Li L. et al., 2019 \\
\hline Oxaliplatin-induced NP & $\operatorname{miR}-15 b$ & $\uparrow$ & $\mathrm{DRG}$ & BACE1 & Ito et al., 2017 \\
\hline $\begin{array}{l}\text { L5 ventral root } \\
\text { transection/paclitaxel-induced } \\
\text { NP }\end{array}$ & miR-500 & $\uparrow$ & Spinal cord horn & GAD67 & Huang et al., 2016 \\
\hline $\mathrm{SCl}$ & miR-23b & $\downarrow$ & Spinal cord & NOX4 & Im et al., 2012 \\
\hline $\mathrm{CCl}$ & $\mathrm{miR}-183$ & $\downarrow$ & Spinal cord horn & MAP3K4/IL-6/IL-1 $\beta / C O X 2$ & Huang and Wang, 2020 \\
\hline SNI & $\operatorname{miR}-21$ & $\uparrow$ & DRG & TLR8 & Zhang et al., 2018c \\
\hline
\end{tabular}

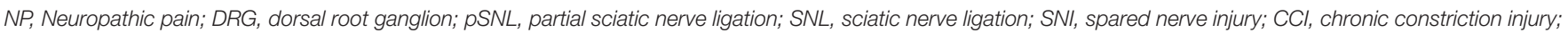

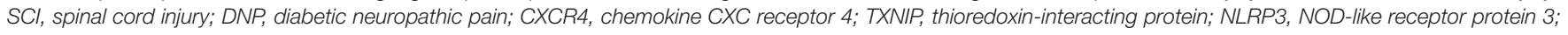

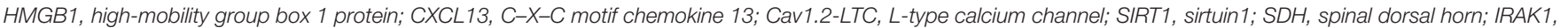

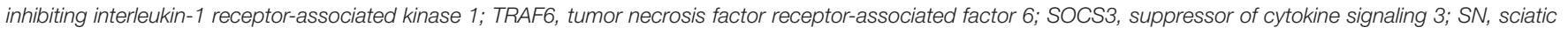

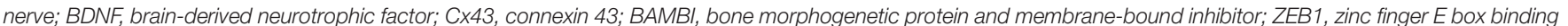

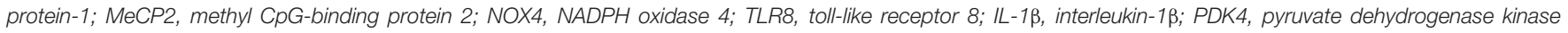

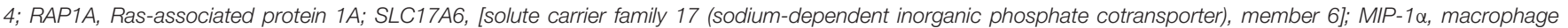

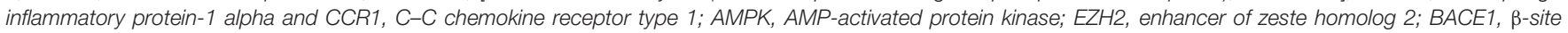
amyloid precursor protein-cleaving enzyme 1. 
In CCI rats, miR-136 could be the target of lncRNA colorectal neoplasia differentially expressed (CRNDE), and the loss of miR136 caused NP by inducing neuroinflammation. In addition, miR-136 also targets IL-6R and regulates its expression. It has been confirmed that increased expression of IL-6R can be induced by CRNDE, and the CRNDE/miR-136/IL-6R axis had a significant effect on NP (Zhang D. et al., 2019). In CCI rats, lncRNA, DGCR5 relieves NP by sponging miR-330-3p and the downstream PDCD4 expression (Peng et al., 2019). In addition, lncRNA $\mathrm{X}$ inactive specific transcript (XIST) can positively regulate NP through regulating the expression of miR-137 and tumor necrosis factor alpha-induced protein 1 (TNFAIP1), which is involved in the regulation of inflammation via activating NF$\mathrm{kB}$ activity (Zhao et al., 2018). Some research concluded that lncRNA, XIST can lead to NP development in rats through the inhibition of miR-544 and activation of STAT3 (Jin et al., 2018). Other research showed that XIST promotes the development of NP by inducing TLR5, which plays a role in the expression of miR-154-5p in CCI rats (Wei et al., 2018). It was found that miR-150 decreased significantly in CCI rats. It was postulated to be the target of XIST and had a negative correlation with XIST. Increased expression of miR-150 can significantly inhibit the expression of neuroinflammatory cytokines. In addition, ZEB1 is the direct target of miR-150. ZEB1 was found to be upregulated in CCI rats. Therefore, the XIST/miR-150/ZEB1 axis can be used as a therapeutic target to mitigate NP (Yan et al., $2018 b)$. In the CCI rat model, the levels of some molecules, such as COX-2, TNF- $\alpha$, interleukin-1 $\beta$ (IL-1 $\beta$ ), and ZEB1 increased. Linc00657 can inhibit these molecules to alleviate NP. Inhibition of miR-136 has the same effect, indicating that ZEB1 is negatively correlated with miR-136 and linc00657 (Shen et al., 2019). LncRNA metastasis-associated lung adenocarcinoma transcript 1 (MALAT1) was significantly upregulated in CCI rats, and miR-206 was significantly downregulated. ZEB2 is the target of miR-206. The upregulation of miR-206 relieves NP caused by ZEB2 overexpression in vivo by inhibiting neuroinflammation. Inhibition of MALAT1 can slow the progression of NP through the miR-206/ZEB2 axis (Chen et al., 2019). In the CCI rat model, the expression of NEAT1 was significantly higher. MiR381 decreased and HMGB1 increased indicating that NEAT1 can regulate the miR-381/HMGB1 axis to slow the progression of NP (Xia et al., 2018). Since natural antisense transcript (NAT) reduces the Nav1.7 current, it is highly likely that lack of NAT increases sodium current. This may lead to an increase in excitability of injury sensitive neurons, which in turn fine-tunes the response to pain stimuli (Koenig et al., 2015).

In SCI rats, inhibition of IncRNA plasmacytoma variant translocation 1 (PVT1) can alleviate NP by upregulating miR-186-5p and downregulating CXCL13/CXCR5 (Zhang P. et al., 2021). SCI rats showed higher nuclear paraspeckle assembly transcript 1 (NEAT1) expression compared to the sham group. Overexpression of NEAT1 enhanced the expression of inflammation factors. NEAT1 targeted and inhibited miR-128-3p, meanwhile miR-128-3p can target aquaporin-4 (AQP4) to regulate its expression (Xian et al., 2021). Compared to the control group, the miR-130a-3p expression was significantly upregulated in the spinal cord lesions of SCI rats. Downregulation of miR-130a-3p increased the expression of IGF-1 and IGF-1R (Yao et al., 2021).

Differential expressions of lncRNAs in NP models and lncRNA-targeted miRNAs or genes have been addressed in detail. LncRNAs play an important role in NP processes not only in neurons but also in non-neuronal cells related to the ion channel, neuroinflammation, purinoceptor, and so on. LncRNAs associated with NP are listed in Table 2.

\section{CIRCULAR RNA IN NEUROPATHIC PAIN}

CircRNAs are produced by natural transcription of genomic DNA, but the bilateral exon transcription is covalently prevented. In most cases, they may be produced by non-covalent splicing. Most of the circRNAs are expressed in the cytoplasm and are very stable, indicating that they may have different functions from those of typical mRNA or lncRNA (Piwecka et al., 2017). CircRNAs are another type of ncRNAs that act as miRNA sponges and modulate gene expression through a circRNA-miRNAmRNA pathway. In the widely used CCI NP models, differentially expressed circRNAs in sham-operated and NP rats were detected by using circRNA microarrays to elucidate the expression of circRNAs in NP (Hansen et al., 2013; Wilusz and Sharp, 2013; Cao et al., 2017).

Zheng et al. (2016) characterized the circRNA named circHIPK3, expressed abundantly from Exon2 of the HIPK3 gene. Silencing the circHIPK3 gene can dramatically inhibit the growth of human cells. Using the luciferase screening experiment, circHIPK3 was found to have 18 potential binding targets and bind nine miRNAs. Specifically, circHIPK3 binds directly to miR124 and inhibits its activity (Zheng et al., 2016). Wang L. et al. (2018) found that circHIPK3 in the serum of DNP patients and the DRG of DNP rats was abundant. In type 2 diabetic patients, the upregulation of circHIPK3 was positively correlated with NP grade. Silencing circHIPK3 can alleviate NP in DNP rats, which is related to neuroinflammation. Further studies into its mechanism of action showed that circHIPK3 interacted with miR-124 and downregulated its expression. The miR-124 inhibitor can reverse the effect of circHIPK3 gene knockout on reducing NP and inhibiting neuroinflammation in DNP rats (Wang L. et al., 2018).

Spinal cord-specific circRNA, circAnksla, increased in both the cytoplasm and the nucleus after SNL. In the cytoplasm, circAnks la promotes the interaction between transcription factor YBX1 and transporter-1, thus facilitating the access of YBX1 to nucleus. The interaction between YBX1 and VEGFB promoter was promoted by specific RNA-DNA interaction. Cytoplasmic circAnksla can also be used as miRNA sponge of miR-324-3p to enhance the translation of VEGFB mRNA. The upregulation of VEGFB stimulated dorsal horn neurons and promoted the pain behavior induced by nerve injury (Zhang S.B. et al., 2019).

In the CCI model, Cai et al. (2020) found that the expression level of ciRS-7 in the SDH was positively associated with the progress of NP; ciRS-7 is involved in the development of NP in part by upregulating autophagy and inflammation. In addition, ciRS-7 sponges to miR-135a-5p to regulate NP. When miR135a-5p expression was inhibited, autophagy and inflammation 
were reduced, and pain was relieved (Cai et al., 2020). In CCI rat models, the expression levels of circ_0005075 were higher. Loss of circ_0005075 could repress neuroinflammation via targeting COX-2, IL-6, and TNF- $\alpha$, while inducing IL-10 in vivo. Additionally, miR-151a-3p was significantly reduced in CCI rats and circ_0005075 reversed the repressive effect of miR$151 \mathrm{a}-3 \mathrm{p}$ on NP. In another study, NOTCH2 has been shown to induce a variety of intracellular responses correlated with NP. In addition, circ_0005075 significantly rescued NOTCH2 expression, which could be repressed by miR-151a-3p (Zhang Y. et al., 2021). Li et al. (2020) found that circZNF609 promoted the expression of inflammatory factors to exacerbate NP progression via the miR-22-3p/Enolase 1 (ENO1) axis in the CCI rat model. It was found that cZRANB1 mediated lysophosphatidic acid receptor 3 (LPAR3) expression via sponging miR-24-3p. MiR$24-3 p$ regulated $\mathrm{Wnt} 5 \mathrm{a} / \mathrm{\beta}$-Catenin signaling levels to promote NP progression via targeting LPAR3 in CCI rats (Wei et al., 2020).

Skin samples collected from patients with postherpetic neuralgia (PHN) were studied using miRNA and circRNA microarray to detect and analyze their expression profiles. Three hundred seventeen miRNAs differed in their expression between PHN-affected skin and normal skin. Only 13 of them showed fold change $>10$ in the PHN skin. Bioinformatics analyses including gene ontology and Kyoto Encyclopedia of Genes and Genomes pathway were conducted to predict the mRNA targets of different miRNAs and also to study the function of these miRNAs. There are 85 pathways that had significant quantities of target miRNAs. This was the first study to research the different expression levels

TABLE 2 | IncRNAs associated with neuropathic pain.

\begin{tabular}{|c|c|c|c|c|c|}
\hline Models & IncRNA & Expression & Tissue & Target gene & References \\
\hline SNL & Kcna2 antisense RNA & $\uparrow$ & DRG & Myeloid zinc finger protein 1 & Zhao et al., 2013 \\
\hline DNP & IncRNA BC168687 & $\uparrow$ & DRG & TRPV1 & Liu C. et al., 2018 \\
\hline DNP & IncRNA BC168687 & $\uparrow$ & SGCs of DRG & $\mathrm{P} 2 \times 7$ & Liu C.L. et al., 2018 \\
\hline DNP & IncRNA NONRATT021972 & $\uparrow$ & $\mathrm{DRG}$ & $\mathrm{P} 2 \times 7$ & Liu et al., 2016 \\
\hline DNP & IncRNA uc $0.48+$ & $\uparrow$ & $\mathrm{DRG}$ & $\mathrm{P} 2 \times 3 / \mathrm{p}-\mathrm{ERK} 1 / 2$ & Wang et al., 2016 \\
\hline DNP & IncRNA NONRATT021972 & $\uparrow$ & Blood & TNF-a & Yu et al., 2017 \\
\hline $\mathrm{SCl}$ & IncRNA PVT1 & $\uparrow$ & Spinal cord & miR-186-5p/CXCL13/CXCR5 & Zhang P. et al., 2021 \\
\hline $\mathrm{SCl}$ & IncRNA NEAT1 & $\uparrow$ & Spinal cord & miR-128-3p/AQP4 & Xian et al., 2021 \\
\hline TN & IncRNA uc $0.48+$ & $\uparrow$ & Trigeminal ganglia & $\mathrm{P} 2 \times 7 / \mathrm{p}-\mathrm{ERK} 1 / 2$ & Xiong et al., 2019 \\
\hline $\mathrm{CCl}$ & IncRNA MRAK009713 & $\uparrow$ & DRG & $\mathrm{P} 2 \times 3$ & Li et al., 2017 \\
\hline SNL & H19 IncRNA & $\uparrow$ & L5 DRG & - & Iwasaki et al., 2019 \\
\hline SNL & IncRNA PKIA-AS1 & $\uparrow$ & Spinal cord & CDK6 & Hu et al., 2019 \\
\hline $\mathrm{CCl}$ & IncRNA CRNDE & $\uparrow$ & L4-L6 dorsal spinal cord & miR-136/IL-6R & Zhang D. et al., 2019 \\
\hline $\mathrm{CCl}$ & IncRNA DGCR5 & $\downarrow$ & L4-L6 dorsal spinal cord & miR-330-3p/PDCD4 & Peng et al., 2019 \\
\hline $\mathrm{CCl}$ & IncRNA XIST & $\uparrow$ & L4-L6 dorsal spinal cord & miR-137/TNFAIP1 & Zhao et al., 2018 \\
\hline $\mathrm{CCl}$ & IncRNA XIST & $\uparrow$ & L4-L6 dorsal spinal cord & miR-544/STAT3 & Jin et al., 2018 \\
\hline $\mathrm{CCl}$ & IncRNA XIST & $\uparrow$ & L4-L6 dorsal spinal cord & miR-154-5p/TLR5 & Wei et al., 2018 \\
\hline $\mathrm{CCl}$ & IncRNA XIST & $\uparrow$ & L4-L6 dorsal spinal cord & miR-150/ZEB1 & Yan et al., 2018b \\
\hline $\mathrm{CCl}$ & IncRNA 00657 & $\uparrow$ & L4-L6 dorsal spinal cord & miR-136/ZEB1 & Shen et al., 2019 \\
\hline $\mathrm{CCl}$ & IncRNA MALAT1 & $\uparrow$ & L4-L6 dorsal spinal cord & miR-206/ZEB2 & Chen et al., 2019 \\
\hline $\mathrm{CCl}$ & linRNA NEAT1 & $\uparrow$ & L4-L6 dorsal spinal cord & miR-381/HMGB1 & Xia et al., 2018 \\
\hline $\mathrm{CCl}$ & SCN9A & $\uparrow$ & L4-L6 ipsilateral DRG & & Koenig et al., 2015 \\
\hline
\end{tabular}

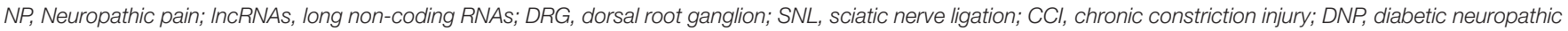

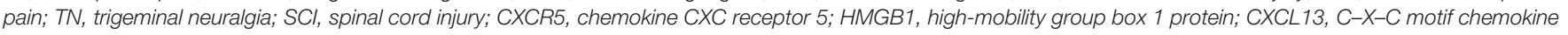

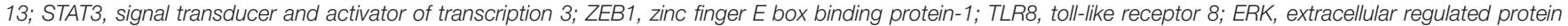

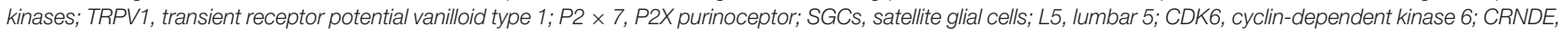
colorectal neoplasia differentially expressed; XIST, X inactive specific transcript; TNFAIP1, tumor necrosis factor alpha-induced protein 1; TNF- $\alpha$, tumor necrosis factor- $\alpha$;

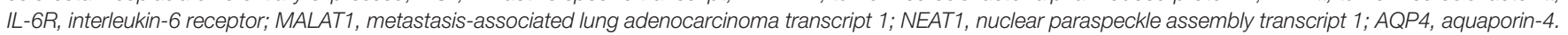

TABLE 3 | CircNAs associated with neuropathic pain.

\begin{tabular}{|c|c|c|c|c|c|}
\hline Models & circRNAs & Expression & Tissue & Target gene & References \\
\hline DNP & circ HIPK3 & $\uparrow$ & $\mathrm{DRG}$ & miR-124 & Wang L. et al., 2018 \\
\hline SNI & circ AnKsla & $\uparrow$ & Spinal cord & VEGFB & Zhang S.B. et al., 2019 \\
\hline $\mathrm{CCl}$ & ciRS-7 & $\uparrow$ & L4-L5 Spinal cord & $\operatorname{miR}-135 a-5 p$ & Cai et al., 2020 \\
\hline $\mathrm{CCl}$ & circ-0005075 & $\uparrow$ & L4-L6 dorsal spinal cord & miR-151a-3p/NOTCH2 & Zhang Y. et al., 2021 \\
\hline $\mathrm{CCl}$ & circ ZNF609 & $\uparrow$ & L4-L6 dorsal spinal cord & miR-22-3p/ENO1 & Li et al., 2020 \\
\hline $\mathrm{CCl}$ & circ ZRANB1 & $\downarrow$ & L4-L6 dorsal spinal cord & Wnt5a/ $\beta$-catenin, miR-24-3p/LPAR3 & Wei et al., 2020 \\
\hline
\end{tabular}

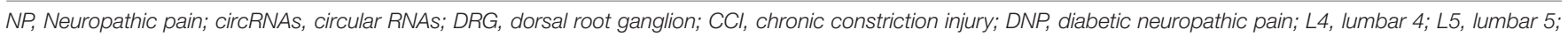
L6, lumbar 6; VEGFB, vascular endothelial growth factor B; ENO1, enolase 1; LPAR3, lysophosphatidic acid receptor 3. 
of miRNA and circRNA in the PHN skin. These abnormally expressed transcripts may be potential therapeutic targets to treat PHN (Cao et al., 2019).

CircRNAs associated with NP are listed in Table 3.

\section{THE LIMITATION IN NEUROPATHIC PAIN RESEARCH}

It has been widely reported that aberrant expression of ncRNAs exists after NP injury, and these differentially expressed ncRNAs are considered to be potential biomarkers for the diagnosis, assessment, treatment, prediction, and prognosis of NP. All these studies indicate that ncRNAs may act as potential biomarkers for clinical utility in patients with NP. Existing studies are limited to cell or animal models. Moreover, there are some differences between in vitro and in vivo experiments, and these differences are unpredictable. Therefore, these data are unpractical to be transformed and applied to the human body, which is still a challenge. Therefore, further studies need to focus on clinical trials to provide more validated evidences that ncRNAs can be used in the diagnosis, treatment, and prognosis of NP ( $\mathrm{Wu} \mathrm{W}$. et al., 2019; Song et al., 2020).

\section{CONCLUSION}

NP is a common disease in clinic, which seriously degenerates the quality of life in patients. The studies about the role of ncRNAs in NP are still at a preliminary stage. In the present

\section{REFERENCES}

Aldrich, B. T., Frakes, E. P., Kasuya, J., Hammond, D. L., and Kitamoto, T. (2009). Changes in expression of sensory organ-specific microRNAs in rat dorsal root ganglia in association with mechanical hypersensitivity induced by spinal nerve ligation. Neuroscience 164, 711-723. doi: 10.1016/j.neuroscience.2009.08.033

Andersen, H. H., Duroux, M., and Gazerani, P. (2014). MicroRNAs as modulators and biomarkers of inflammatory and neuropathic pain conditions. Neurobiol. Dis. 71, 159-168. doi: 10.1016/j.nbd.2014.08.003

Bali, K. K., and Kuner, R. (2014). Noncoding RNAs: key molecules in understanding and treating pain. Trends Mol. Med. 20, 437-448. doi: 10.1016/j. molmed.2014.05.006

Bali, K. K., Selvaraj, D., Satagopam, V. P., Lu, J., Schneider, R., and Kuner, R. (2013). Genome-wide identification and functional analyses of microRNA signatures associated with cancer pain. EMBO Mol. Med. 5, 1740-1758. doi: 10.1002/ emmm. 201302797

Cai, W., Zhang, Y., and Su, Z. (2020). ciRS-7 targeting miR-135a-5p promotes neuropathic pain in CCI rats via inflammation and autophagy. Gene 736:144386. doi: 10.1016/j.gene.2020.144386

Cao, S., Deng, W., Li, Y., Qin, B., Zhang, L., Yu, S., et al. (2017). Chronic constriction injury of sciatic nerve changes circular RNA expression in rat spinal dorsal horn. J. Pain Res. 10, 1687-1696. doi: 10.2147/JPR.S139592

Cao, S., Zhang, D., Yuan, J., Liu, C., Zhou, W., Zhang, L., et al. (2019). MicroRNA and circular RNA expression in affected skin of patients with postherpetic neuralgia. J. Pain Res. 12, 2905-2913. doi: 10.2147/JPR.S221615

Chen, Z. L., Liu, J. Y., Wang, F., and Jing, X. (2019). Suppression of MALAT1 ameliorates chronic constriction injury-induced neuropathic pain in rats via modulating miR-206 and ZEB2. J. Cell. Physiol. 234, 15647-15653. doi: 10.1002/ jcp. 28213

Du, H., Liu, Z., Tan, X., Ma, Y., and Gong, Q. (2019). Identification of the genomewide expression patterns of long non-coding RNAs and mRNAs in mice with study, some ncRNAs in the damaged peripheral nerve, DRG, and $\mathrm{SDH}$ may have a significant effect on the NP, but the detailed mechanisms of how the majority of these ncRNAs contribute to this disorder need more explorations. The aforementioned studies suggest that ncRNAs are endogenous promoters of chronic pain in the peripheral and central nervous system. ncRNAs may have potential to be used as biomarkers and new drug targets to treat NP. ncRNAs are supposed to have multiple and specific downstream targets due to their characters. However, the functions and mechanisms of these ncRNAs are needed to be elucidated in further studies.

\section{AUTHOR CONTRIBUTIONS}

WT designed the review and provided financial support. $\mathrm{CH}$, $\mathrm{MH}$, and QX collected the data from publications. $\mathrm{CH}$ developed the database, wrote the manuscript, and edited the final text. All authors read and approved the final manuscript.

\section{FUNDING}

This present study was supported by the National Natural Science Foundation of China (grant no. 81673741) to WT and (grant no. 81803859) to QX, the Natural Science Foundation of Jiangsu Province (grant no. BK20181096) to QX, Postgraduate Research and Practice Innovation Program of Jiangsu Province (grant no. SJCX21_0783) to CH.

streptozotocin-induced diabetic neuropathic pain. Neuroscience 402, 90-103. doi: 10.1016/j.neuroscience.2018.12.040

Favereaux, A., Thoumine, O., Bouali-Benazzouz, R., Roques, V., Papon, M. A., Salam, S. A., et al. (2011). Bidirectional integrative regulation of Cav1.2 calcium channel by microRNA miR-103: role in pain. EMBO J. 30, 3830-3841. doi: 10.1038/emboj.2011.249

Gao, P., Zeng, X., Zhang, L., Wang, L., Shen, L. L., Hou, Y. Y., et al. (2021). Overexpression of miR-378 alleviates chronic sciatic nerve injury by targeting EZH2. Neurochem. Res. 46, 3213-3221. doi: 10.1007/s11064-021-03 424-9

Gaudet, A. D., Fonken, L. K., Watkins, L. R., Nelson, R. J., and Popovich, P. G. (2018). MicroRNAs: roles in regulating neuroinflammation. Neuroscientist 24, 221-245. doi: 10.1177/1073858417721150

Gilron, I., and Dickenson, A. H. (2014). Emerging drugs for neuropathic pain. Expert Opin. Emerg. Drugs 19, 329-341. doi: 10.1517/14728214.2014.915025

Hansen, T. B., Jensen, T. I., Clausen, B. H., Bramsen, J. B., Finsen, B., Damgaard, C. K., et al. (2013). Natural RNA circles function as efficient microRNA sponges. Nature 495, 384-388. doi: 10.1038/nature11993

Heyn, J., Luchting, B., Hinske, L. C., Hubner, M., Azad, S. C., and Kreth, S. (2016). miR-124a and miR-155 enhance differentiation of regulatory $\mathrm{T}$ cells in patients with neuropathic pain. J. Neuroinflammation 13:248. doi: 10.1186/s12974-0160712-6

Holmes, D. (2016). The pain drain. Nature 535, S2-S3. doi: 10.1038/535S2a

Hori, N., Narita, M., Yamashita, A., Horiuchi, H., Hamada, Y., Kondo, T., et al. (2016). Changes in the expression of IL-6-mediated microRNAs in the dorsal root ganglion under neuropathic pain in mice. Synapse 70, 317-324. doi: 10. 1002/syn. 21902

Hu, J. Z., Rong, Z. J., Li, M., Li, P., Jiang, L. Y., Luo, Z. X., et al. (2019). Silencing of lncRNA PKIA-AS1 attenuates spinal nerve ligation-induced neuropathic pain through epigenetic downregulation of CDK6 expression. Front. Cell. Neurosci. 13:50. doi: 10.3389/fncel.2019.00050 
Huang, L., and Wang, L. (2020). Upregulation of miR-183 represses neuropathic pain through inhibiton of MAP3K4 in CCI rat models. J. Cell. Physiol. 235, 3815-3822. doi: $10.1002 /$ jcp.29276

Huang, Z. Z., Wei, J. Y., Ou-Yang, H. D., Li, D., Xu, T., Wu, S. L., et al. (2016). mir-500-Mediated GAD67 downregulation contributes to neuropathic pain. J. Neurosci. 36, 6321-6331. doi: 10.1523/JNEUROSCI.0646-16.2016

Im, Y. B., Jee, M. K., Choi, J. I., Cho, H. T., Kwon, O. H., and Kang, S. K. (2012). Molecular targeting of NOX4 for neuropathic pain after traumatic injury of the spinal cord. Cell Death Dis. 3:e426. doi: 10.1038/cddis.2012.168

Ito, N., Sakai, A., Miyake, N., Maruyama, M., Iwasaki, H., Miyake, K., et al. (2017). miR-15b mediates oxaliplatin-induced chronic neuropathic pain through BACE1 down-regulation. Br. J. Pharmacol. 174, 386-395.

Iwasaki, H., Sakai, A., Maruyama, M., Ito, T., Sakamoto, A., and Suzuki, H. (2019). Increased H19 long non-coding RNA expression in Schwann cells in peripheral neuropathic pain. J. Nippon Med. Sch. 86, 215-221. doi: 10.1272/jnms.JNMS. 2018_86-402

Jiang, B. C., Cao, D. L., Zhang, X., Zhang, Z. J., He, L. N., Li, C. H., et al. (2016). CXCL13 drives spinal astrocyte activation and neuropathic pain via CXCR5. J. Clin. Invest. 126, 745-761. doi: 10.1172/JCI81950

Jin, H., Du, X. J., Zhao, Y., and Xia, D. L. (2018). XIST/miR-544 axis induces neuropathic pain by activating STAT3 in a rat model. J. Cell. Physiol. 233, 5847-5855. doi: $10.1002 /$ jcp. 26376

Jin, Y., Xu, L., and Xu, Y. (2021). Effect of intrathecal injection of miRNA-138 on neuropathic pain in rats undergoing partial sciatic nerve ligation and its underlying mechanism. Ann. Palliat. Med. 10, 6873-6882. doi: 10.21037/apm21-669

Kalpachidou, T., Kummer, K. K., and Kress, M. (2020). Non-coding RNAs in neuropathic pain. Neuronal Signal. 4:NS20190099. doi: 10.1042/NS20190099

Koenig, J., Werdehausen, R., Linley, J. E., Habib, A. M., Vernon, J., Lolignier, S., et al. (2015). Regulation of Nav1.7: a conserved SCN9A natural antisense transcript expressed in dorsal root ganglia. PLoS One 10:e0128830.

Leinders, M., Uceyler, N., Pritchard, R. A., Sommer, C., and Sorkin, L. S. (2016). Increased miR-132-3p expression is associated with chronic neuropathic pain. Exp. Neurol. 283(Pt A), 276-286. doi: 10.1016/j.expneurol.2016.06.025

Li, G., Jiang, H., Zheng, C., Zhu, G., Xu, Y., Sheng, X., et al. (2017). Long noncoding RNA MRAK009713 is a novel regulator of neuropathic pain in rats. Pain 158, 2042-2052. doi: 10.1097/j.pain.0000000000001013

Li, L., Luo, Y., Zhang, Y., Wei, M., Zhang, M., Liu, H., et al. (2020). CircZNF609 aggravates neuropathic pain via miR-22-3p/ENO1 axis in CCI rat models. Gene 763:145069. doi: 10.1016/j.gene.2020.145069

Li, L., Shao, J., Wang, J., Liu, Y., Zhang, Y., Zhang, M., et al. (2019). MiR-30b$5 p$ attenuates oxaliplatin-induced peripheral neuropathic pain through the voltage-gated sodium channel Nav1.6 in rats. Neuropharmacology 153, 111-120. doi: 10.1016/j.neuropharm.2019.04.024

Li, L., and Zhao, G. (2016). Downregulation of microRNA-218 relieves neuropathic pain by regulating suppressor of cytokine signaling 3. Int. J. Mol. Med. 37, 851-858. doi: 10.3892/ijmm.2016.2455

Li, X., Wang, S., Yang, X., and Chu, H. (2021). miR1423p targets AC9 to regulate sciatic nerve injuryinduced neuropathic pain by regulating the cAMP/AMPK signalling pathway. Int. J. Mol. Med. 47, 561-572. doi: 10.3892/ijmm.2020.4824

Li, Z., Li, X., Chen, X., Li, S., Ho, I. H. T., Liu, X., et al. (2019). Emerging roles of long non-coding RNAs in neuropathic pain. Cell Prolif. 52:e12528.

Liu, C., Li, C., Deng, Z., Du, E., and Xu, C. (2018). Long non-coding RNA BC168687 is involved in TRPV1-mediated diabetic neuropathic pain in rats. Neuroscience 374, 214-222. doi: 10.1016/j.neuroscience.2018.01.049

Liu, C. L., Deng, Z. Y., Du, E. R., and Xu, C. S. (2018). Long noncoding RNA BC168687 small interfering RNA reduces high glucose and high free fatty acidinduced expression of P2X7 receptors in satellite glial cells. Mol. Med. Rep. 17, 5851-5859. doi: 10.3892/mmr.2018.8601

Liu, S., Zou, L., Xie, J., Xie, W., Wen, S., Xie, Q., et al. (2016). LncRNA NONRATT021972 siRNA regulates neuropathic pain behaviors in type 2 diabetic rats through the $\mathrm{P} 2 \mathrm{X} 7$ receptor in dorsal root ganglia. Mol. Brain 9:44.

Liu, Z., Liang, Y., Wang, H., Lu, Z., Chen, J., Huang, Q., et al. (2017). LncRNA expression in the spinal cord modulated by minocycline in a mouse model of spared nerve injury. J. Pain Res. 10, 2503-2514. doi: 10.2147/JPR.S147055

Lu, Y., Cao, D. L., Jiang, B. C., Yang, T., and Gao, Y. J. (2015). MicroRNA-146a$5 \mathrm{p}$ attenuates neuropathic pain via suppressing TRAF6 signaling in the spinal cord. Brain Behav. Immun. 49, 119-129. doi: 10.1016/j.bbi.2015.04.018
Lutz, B. M., Bekker, A., and Tao, Y. X. (2014). Noncoding RNAs: new players in chronic pain. Anesthesiology 121, 409-417. doi: 10.1097/ALN. 0000000000000265

Neumann, E., Hermanns, H., Barthel, F., Werdehausen, R., and Brandenburger, T. (2015). Expression changes of microRNA-1 and its targets Connexin 43 and brain-derived neurotrophic factor in the peripheral nervous system of chronic neuropathic rats. Mol. Pain 11:39. doi: 10.1186/s12990-015-0045-y

Pan, Z., Shan, Q., Gu, P., Wang, X. M., Tai, L. W., Sun, M., et al. (2018). miRNA23a/CXCR4 regulates neuropathic pain via directly targeting TXNIP/NLRP3 inflammasome axis. J. Neuroinflammation 15:29. doi: 10.1186/s12974-0181073-0

Pan, Z., Zhang, M., Ma, T., Xue, Z. Y., Li, G. F., Hao, L. Y., et al. (2016). Hydroxymethylation of microRNA-365-3p regulates nociceptive behaviors via Kcnh2. J. Neurosci. 36, 2769-2781. doi: 10.1523/JNEUROSCI.3474-15.2016

Pan, Z., Zhu, L. J., Li, Y. Q., Hao, L. Y., Yin, C., Yang, J. X., et al. (2014). Epigenetic modification of spinal miR-219 expression regulates chronic inflammation pain by targeting CaMKIIgamma. J. Neurosci. 34, 9476-9483. doi: 10.1523/ JNEUROSCI.5346-13.2014

Park, C. K., Xu, Z. Z., Berta, T., Han, Q., Chen, G., Liu, X. J., et al. (2014). Extracellular microRNAs activate nociceptor neurons to elicit pain via TLR7 and TRPA1. Neuron 82, 47-54. doi: 10.1016/j.neuron.2014.02.011

Peng, C., Li, L., Zhang, M. D., Bengtsson Gonzales, C., Parisien, M., Belfer, I., et al. (2017). miR-183 cluster scales mechanical pain sensitivity by regulating basal and neuropathic pain genes. Science 356, 1168-1171. doi: 10.1126/science. aam7671

Peng, C., Zhang, C., Su, Z., and Lin, D. (2019). DGCR5 attenuates neuropathic pain through sponging miR-330-3p and regulating PDCD4 in CCI rat models. J. Cell. Physiol. 234, 7292-7300. doi: 10.1002/jcp.27487

Piwecka, M., Glazar, P., Hernandez-Miranda, L. R., Memczak, S., Wolf, S. A., Rybak-Wolf, A., et al. (2017). Loss of a mammalian circular RNA locus causes miRNA deregulation and affects brain function. Science 357:eaam8526. doi: 10.1126/science.aam8526

Plassais, J., Lagoutte, L., Correard, S., Paradis, M., Guaguere, E., Hedan, B., et al. (2016). A point mutation in a lincRNA upstream of GDNF is associated to a canine insensitivity to pain: a spontaneous model for human sensory neuropathies. PLoS Genet. 12:e1006482. doi: 10.1371/journal.pgen.1006482

Sakai, A., Saitow, F., Maruyama, M., Miyake, N., Miyake, K., Shimada, T., et al. (2017). MicroRNA cluster miR-17-92 regulates multiple functionally related voltage-gated potassium channels in chronic neuropathic pain. Nat. Commun. 8:16079. doi: 10.1038/ncomms16079

Sakai, A., Saitow, F., Miyake, N., Miyake, K., Shimada, T., and Suzuki, H. (2013). miR-7a alleviates the maintenance of neuropathic pain through regulation of neuronal excitability. Brain 136(Pt 9), 2738-2750. doi: 10.1093/brain/awt191

Shao, J., Wang, J., Huang, J., Liu, C., Pan, Y., Guo, Q., et al. (2018). Identification of IncRNA expression profiles and ceRNA analysis in the spinal cord of morphine-tolerant rats. Mol. Brain 11:21. doi: 10.1186/s13041-018-0365-8

Shen, F., Zheng, H., Zhou, L., Li, W., Zhang, Y., and Xu, X. (2019). LINC00657 expedites neuropathic pain development by modulating miR-136/ZEB1 axis in a rat model. J. Cell. Biochem. 120, 1000-1010. doi: 10.1002/jcb.27466

Song, G., Yang, Z., Guo, J., Zheng, Y., Su, X., and Wang, X. (2020). Interactions among lncRNAs/circRNAs, miRNAs, and mRNAs in neuropathic pain. Neurotherapeutics 17, 917-931. doi: 10.1007/s13311-020-00881-y

Su, S., Shao, J., Zhao, Q., Ren, X., Cai, W., Li, L., et al. (2017). MiR-30b attenuates neuropathic pain by regulating voltage-gated sodium channel Nav1.3 in rats. Front. Mol. Neurosci. 10:126. doi: 10.3389/fnmol.2017.00126

Sun, Y., Li, X. Q., Sahbaie, P., Shi, X. Y., Li, W. W., Liang, D. Y., et al. (2012). miR-203 regulates nociceptive sensitization after incision by controlling phospholipase A2 activating protein expression. Anesthesiology 117, 626-638. doi: 10.1097/ALN.0b013e31826571aa

Tramullas, M., Frances, R., de la Fuente, R., Velategui, S., Carcelen, M., Garcia, R., et al. (2018). MicroRNA-30c-5p modulates neuropathic pain in rodents. Sci. Transl. Med. 10:eaao6299. doi: 10.1126/scitranslmed.aao6299

Vieira, A. S., Dogini, D. B., and Lopes-Cendes, I. (2018). Role of non-coding RNAs in non-aging-related neurological disorders. Braz. J. Med. Biol. Res. 51:e7566. doi: 10.1590/1414-431X20187566

von Hehn, C. A., Baron, R., and Woolf, C. J. (2012). Deconstructing the neuropathic pain phenotype to reveal neural mechanisms. Neuron 73, 638-652. doi: $10.1016 /$ j.neuron.2012.02.008 
Wan, L., Su, Z., Li, F., Gao, P., and Zhang, X. (2021). MiR-122-5p suppresses neuropathic pain development by targeting PDK4. Neurochem. Res. 46, 957963. doi: 10.1007/s11064-020-03213-w

Wang, L., Luo, T., Bao, Z., Li, Y., and Bu, W. (2018). Intrathecal circHIPK3 shRNA alleviates neuropathic pain in diabetic rats. Biochem. Biophys. Res. Commun. 505, 644-650. doi: 10.1016/j.bbrc.2018.09.158

Wang, S., Xu, H., Zou, L., Xie, J., Wu, H., Wu, B., et al. (2016). LncRNA uc.48+ is involved in diabetic neuropathic pain mediated by the $\mathrm{P} 2 \mathrm{X} 3$ receptor in the dorsal root ganglia. Purinergic Signal. 12, 139-148.

Wang, Z., Liu, F., Wei, M., Qiu, Y., Ma, C., Shen, L., et al. (2018). Chronic constriction injury-induced microRNA-146a-5p alleviates neuropathic pain through suppression of IRAK1/TRAF6 signaling pathway. J. Neuroinflammation 15:179. doi: 10.1186/s12974-018-1215-4

Wei, M., Li, L., Zhang, Y., Zhang, M., and Su, Z. (2020). Downregulated circular RNA zRANB1 mediates Wnt5a/beta-Catenin signaling to promote neuropathic pain via miR-24-3p/LPAR3 axis in CCI rat models. Gene 761:145038. doi: 10. 1016/j.gene.2020.145038

Wei, M., Li, L., Zhang, Y., Zhang, Z. J., Liu, H. L., and Bao, H. G. (2018). LncRNA $\mathrm{X}$ inactive specific transcript contributes to neuropathic pain development by sponging miR-154-5p via inducing toll-like receptor 5 in CCI rat models. J. Cell. Biochem. 120, 1271-1281. doi: 10.1002/jcb.27088

Widerstrom-Noga, E. (2017). Neuropathic pain and spinal cord injury: phenotypes and pharmacological management. Drugs 77, 967-984.

Wilusz, J. E., and Sharp, P. A. (2013). Molecular biology. A circuitous route to noncoding RNA. Science 340, 440-441. doi: 10.1126/science.1238522

Wu, B., Guo, Y., Chen, Q., Xiong, Q., and Min, S. (2019). MicroRNA-193a downregulates HMGB1 to alleviate diabetic neuropathic pain in a mouse model. Neuroimmunomodulation 26, 250-257. doi: 10.1159/000503325

Wu, W., Ji, X., and Zhao, Y. (2019). Emerging roles of long non-coding RNAs in chronic neuropathic pain. Front. Neurosci. 13:1097. doi: 10.3389/fnins.2019. 01097

Wu, X., Wang, X., Yin, Y., Zhu, L., Zhang, F., and Yang, J. (2021). Investigation of the role of miR-221 in diabetic peripheral neuropathy and related molecular mechanisms. Adv. Clin. Exp. Med. 30, 623-632. doi: 10.17219/acem/131217

Wu, Y., Gu, Y., and Shi, B. (2020). miR-590-3p alleviates diabetic peripheral neuropathic pain by targeting RAP1A and suppressing infiltration by the T cells. Acta Biochim. Pol. 67, 587-593. doi: 10.18388/abp.2020_5451

Xia, L., Zhang, Y., and Dong, T. (2016). Inhibition of microRNA-221 alleviates neuropathic pain through targeting suppressor of cytokine signaling 1. J. Mol. Neurosci. 59, 411-420. doi: 10.1007/s12031-016-0748-1

Xia, L. X., Ke, C., and Lu, J. M. (2018). NEAT1 contributes to neuropathic pain development through targeting miR-381/HMGB1 axis in CCI rat models. J. Cell. Physiol. 233, 7103-7111. doi: 10.1002/jcp.26526

Xian, S., Ding, R., Li, M., and Chen, F. (2021). LncRNA NEAT1/miR-128-3p/AQP4 axis regulating spinal cord injury-induced neuropathic pain progression. J. Neuroimmunol. 351:577457. doi: 10.1016/j.jneuroim.2020.577457

Xie, X., Ma, L., Xi, K., Zhang, W., and Fan, D. (2017). MicroRNA-183 suppresses neuropathic pain and expression of AMPA receptors by targeting mTOR/VEGF signaling pathway. Cell. Physiol. Biochem. 41, 181-192. doi: 10.1159/000455987

Xie, X. J., Ma, L. G., Xi, K., Fan, D. M., Li, J. G., Zhang, Q., et al. (2017). Effects of microRNA-223 on morphine analgesic tolerance by targeting NLRP3 in a rat model of neuropathic pain. Mol. Pain 13:1744806917706582. doi: 10.1177/ 1744806917706582

Xiong, W., Tan, M., Tong, Z., Yin, C., He, L., Liu, L., et al. (2019). Effects of long non-coding RNA uc.48+ on pain transmission in trigeminal neuralgia. Brain Res. Bull. 147, 92-100. doi: 10.1016/j.brainresbull.2019.02.009

Xu, L., Wang, Q., Jiang, W., Yu, S., and Zhang, S. (2019). MiR-34c ameliorates neuropathic pain by targeting NLRP3 in a mouse model of chronic constriction injury. Neuroscience 399, 125-134. doi: 10.1016/j.neuroscience.2018.12.030

Yan, T., Zhang, F., Sun, C., Sun, J., Wang, Y., Xu, X., et al. (2018a). miR-325p-mediated Dusp5 downregulation contributes to neuropathic pain. Biochem. Biophys. Res. Commun. 495, 506-511. doi: 10.1016/j.bbrc.2017.11.013

Yan, X. T., Lu, J. M., Wang, Y., Cheng, X. L., He, X. H., Zheng, W. Z., et al. (2018b). XIST accelerates neuropathic pain progression through regulation of miR-150 and ZEB1 in CCI rat models. J. Cell. Physiol. 233, 6098-6106. doi: $10.1002 /$ jcp. 26453

Yan, X. T., Zhao, Y., Cheng, X. L., He, X. H., Wang, Y., Zheng, W. Z., et al. (2018c). Inhibition of miR-200b/miR-429 contributes to neuropathic pain development through targeting zinc finger E box binding protein-1. J. Cell. Physiol. 233, 4815-4824. doi: 10.1002/jcp.26284
Yan, X. T., Ji, L. J., Wang, Z., Wu, X., Wang, Q., Sun, S., et al. (2017). MicroRNA-93 alleviates neuropathic pain through targeting signal transducer and activator of transcription 3. Int. Immunopharmacol. 46, 156-162.

Yang, D., Yang, Q., Wei, X., Liu, Y., Ma, D., Li, J., et al. (2017). The role of miR$190 \mathrm{a}-5 \mathrm{p}$ contributes to diabetic neuropathic pain via targeting SLC17A6. J. Pain Res. 10, 2395-2403. doi: 10.2147/JPR.S133755

Yao, L., Guo, Y., Wang, L., Li, G., Qian, X., Zhang, J., et al. (2021). Knockdown of miR-130a-3p alleviates spinal cord injury induced neuropathic pain by activating IGF-1/IGF-1R pathway. J. Neuroimmunol. 351:577458. doi: 10.1016/ j.jneuroim.2020.577458

Yu, W., Zhao, G. Q., Cao, R. J., Zhu, Z. H., and Li, K. (2017). LncRNA NONRATT021972 was associated with neuropathic pain scoring in patients with type 2 diabetes. Behav. Neurol. 2017:2941297. doi: 10.1155/2017/2941297

Zhan, L. Y., Lei, S. Q., Zhang, B. H., Li, W. L., Wang, H. X., Zhao, B., et al. (2018). Overexpression of miR-381 relieves neuropathic pain development via targeting HMGB1 and CXCR4. Biomed. Pharmacother. 107, 818-823.

Zhang, D., Mou, J. Y., Wang, F., Liu, J., and Hu, X. (2019). CRNDE enhances neuropathic pain via modulating miR-136/IL6R axis in CCI rat models. J. Cell. Physiol. 234, 22234-22241. doi: 10.1002/jcp.28790

Zhang, P., Sun, H., and Ji, Z. (2021). Downregulating lncRNA PVT1 relieves astrocyte overactivation induced neuropathic pain through targeting miR186-5p/CXCL13/CXCR5 Axis. Neurochem. Res. 46, 1457-1469. doi: 10.1007/ s11064-021-03287-0

Zhang, R., Huang, M., Cao, Z., Qi, J., Qiu, Z., and Chiang, L. Y. (2015). MeCP2 plays an analgesic role in pain transmission through regulating CREB/miR-132 pathway. Mol. Pain 11:19. doi: 10.1186/s12990-015-0015-4

Zhang, S. B., Lin, S. Y., Liu, M., Liu, C. C., Ding, H. H., Sun, Y., et al. (2019). CircAnks1a in the spinal cord regulates hypersensitivity in a rodent model of neuropathic pain. Nat. Commun. 10:4119. doi: 10.1038/s41467-019-12049-0

Zhang, Y., Gao, T., Li, X., Wen, C. C., Yan, X. T., Peng, C., et al. (2021). Circ_0005075 targeting miR-151a-3p promotes neuropathic pain in CCI rats via inducing NOTCH2 expression. Gene 767:145079.

Zhang, Y., Su, Z., Liu, H. L., Li, L., Wei, M., Ge, D. J., et al. (2018a). Effects of miR$26 a-5 p$ on neuropathic pain development by targeting MAPK6 in in CCI rat models. Biomed. Pharmacother. 107, 644-649.

Zhang, Z. J., Guo, J. S., Li, S. S., Wu, X. B., Cao, D. L., Jiang, B. C., et al. (2018c). TLR 8 and its endogenous ligand miR-21 contribute to neuropathic pain in murine DRG. J. Exp. Med. 215, 3019-3037. doi: 10.1084/jem.20180800

Zhang, Y., Tao, Y., and Liao, Q. (2018b). Long noncoding RNA: a crosslink in biological regulatory network. Brief. Bioinform. 19, 930-945. doi: 10.1093/bib/ bbx042

Zhao, X., Tang, Z., Zhang, H., Atianjoh, F. E., Zhao, J. Y., Liang, L., et al. (2013). A long noncoding RNA contributes to neuropathic pain by silencing Kcna2 in primary afferent neurons. Nat. Neurosci. 16, 1024-1031. doi: 10.1038/nn.3438

Zhao, Y., Li, S., Xia, N., Shi, Y., and Zhao, C. M. (2018). Effects of XIST/miR-137 axis on neuropathic pain by targeting TNFAIP1 in a rat model. J. Cell. Physiol. 233, 4307-4316. doi: 10.1002/jcp.26254

Zheng, Q., Bao, C., Guo, W., Li, S., Chen, J., Chen, B., et al. (2016). Circular RNA profiling reveals an abundant circHIPK3 that regulates cell growth by sponging multiple miRNAs. Nat. Commun. 7:11215. doi: 10.1038/ncomms11215

Zhu, J., Yang, J., and $\mathrm{Xu}$, J. (2021). miR-223 inhibits the polarization and recruitment of macrophages via NLRP3/IL-1beta pathway to meliorate neuropathic pain. Pain Res. Manag. 2021:6674028. doi: 10.1155/2021/6674028

Conflict of Interest: The authors declare that the research was conducted in the absence of any commercial or financial relationships that could be construed as a potential conflict of interest.

Publisher's Note: All claims expressed in this article are solely those of the authors and do not necessarily represent those of their affiliated organizations, or those of the publisher, the editors and the reviewers. Any product that may be evaluated in this article, or claim that may be made by its manufacturer, is not guaranteed or endorsed by the publisher.

Copyright (c) $2021 \mathrm{Hu}, \mathrm{He}, \mathrm{Xu}$ and Tian. This is an open-access article distributed under the terms of the Creative Commons Attribution License (CC BY). The use, distribution or reproduction in other forums is permitted, provided the original author(s) and the copyright owner(s) are credited and that the original publication in this journal is cited, in accordance with accepted academic practice. No use, distribution or reproduction is permitted which does not comply with these terms. 